\title{
Experimental Evaluation of Techniques Designed to Reduce Vibration Simulation Test Time
}

\author{
Kyle Dunno \\ Clemson University \\ kdunno@,clemson.edu
}

\begin{abstract}
Performing accurate vehicle vibration simulations is imperative to understanding the adequacy of a packaged product's ability to withstand the rigors of transportation. Over the past several years, various vehicle vibration analysis techniques have been proposed in order to provide better correlation to actual field shipments. Some of these new techniques allow for the vibration data collected to be analyzed in a manner that reduces the duration of the laboratory vibration simulation. This paper discusses the discoveries made when an independent laboratory utilized these techniques to reduce test durations for vertical vibration simulation. A field data recorder was employed to collect and record over-the-road vibration of a fully loaded steel spring truck traveling over interstates and highways. The collected data was analyzed independently for each of the different simulation techniques and the resulting simulations were conducted using a servo-hydraulic vibration table. This paper is unique in it evaluates the simulation techniques using actual packaged products, and by use of a damage assessment tool, determines how well the techniques correlated with actual field study results. The three packaged products used for these evaluations were as follows: top-mount refrigerator, electric hedge trimmer, and gas powered pressure washer. Results indicate it is possible to reduce the vibration test durations during vertical vibration simulation, while still correlating with results collected from field studies.
\end{abstract}

Key Words: Vibration Testing, Random Vibration, Package Testing, Vibration Techniques

\subsection{INTRODUCTION}

Vibration is the most common hazard occurring to all packaged products as they pass through any distribution cycle. Since vibration occurs to all packaged products, it is necessary to evaluate packaged products to determine if the both the package and product are able to survive and be functional at the end of a given shipment. Other hazards are of importance as well, such as shock, compression, and environmental, but the one hazard all packaged products will encounter is vibration [1]. 
Vehicle vibration is the result of a vehicle traveling over a road surface exciting the vehicle as it travels. The vehicles response to these excitations is dependent on a number of factors such as road surface, suspension characteristics, and the vehicle's velocity and payload. Due to the numerous parameters and variables affecting the response, there is inherent variability in vibration intensity during a shipment and also in the intensity of vibration between shipments [2].

Currently, three methods are employed to simulate vibration in a laboratory. These can be separated into the following categories: repetitive shock (fixed displacement), random vibration, and multi-axis vibration. Repetitive shock is commonly performed on a mechanical shaker table where a system of cams moves the platform in a circular motion. Although while useful and very widely conducted, repetitive shock testing is not generally regarded as a good simulation of actual transport vibration [3]. Random vibration is the most common method used to simulate transport vibration. A technique used to produce random vibration PSD profiles is fast Fourier transformation (FFT). Snapshots of the amplitude-time history are taken and produce amplitude frequency spectra at a rate dependent on the frequency resolution. Each spectra is then averaged to produce an average spectrum often presented in Power Spectral Density $\left(\mathrm{g}^{2} / \mathrm{Hz}\right)$. Because actual vehicle vibration is not restricted to only vertical motion, multi-axis vibration testing is gaining exposure due to its ability to excite in other axes of interest. Excitations from a multi-axis simulation can provide a better understanding of the influence of lateral, longitudinal, pitch, roll, and yaw movements on the packaging response [4].

The goal of vibration testing in a laboratory is to closely replicate the actual vibration expe- rienced in the shipping environment. Ideally, test laboratories want to accomplish this in the shortest amount of time as possible. Reproducing shorter duration shipments works well in a laboratory setting, but what if the trip of interest is 15 or 20 hours? For most test laboratories, performing a 15 or 20 hour vibration test is not practical. For these cases, reducing the test duration is an appealing option. This paper focuses on the following test methods for reducing the duration of vibration test simulations: Time Compression (Accelerated), Constructed Probability Analysis, and Non-Stationary and Non-Gaussian. These techniques have been introduced at past ISTA Transpack Forums as having the ability to accurately correlate with the vibration occurring during actual over-the-road shipments [5]. This project used packaged products to determine if these techniques can be employed to properly reduce the test time of a vibration simulation while still reproducing actual field results.

\subsection{Time Compression (Accelerated) Vibration}

Many of the published vibration profiles outlined in ISTA and ASTM are time compressed in order to decrease the overall test time required for laboratory testing [6] [7]. As a general rule of thumb, the profile should not exceed a compression of more than $5: 1$; where five is the number of actual truck hours and one is the number of laboratory test hours. The commonly used equation (equation 1), displays the method for accelerating, or time compressing a vibration test. When time compressing a profile the shape of the given profile remains unchanged; it simply gets increased in overall intensity (Grms) to permit compression of the testing time [3]. 
Eq. 1: $\quad I_{T}=I_{O} \sqrt{\frac{T_{O}}{T_{T}}}$

Where $I_{T}=$ overall intensity of the test lab profile (expressed in Grms)

$I_{0}=$ field-measured intensity of transport profile (in Grms)

$\mathrm{T}_{0}=$ time duration of the transport vibration

$\mathrm{T}_{\mathrm{T}}=$ the test time

\subsection{Constructed Probability Analysis}

The Constructed Probability Analysis technique breaks the vibration data into different levels based on the probability of occurrence. The levels examined and used by Root were as follows: $50 \%$, $80 \%, 90 \%, 95 \%, 99 \%$, and $100 \%$ [8]. The different percentiles produce separate PSD profiles which are run in a sequence to evaluate the vibration data recorded. The PSD profiles generated do not represent any one actual event, but rather are a statistical representation of the overall energy which occurred within that percentile range. The separate PSD profiles are run for varying lengths of time, which are dependent on the number of events occurring for that percentile range, and the length of each event recorded.

\subsection{Synthesis of Non-stationary and Non- Gaussian}

Stationary random vibration is defined by statistical properties that do not change with time. In the transportation environment, such a condition might be approximated by a truck traveling long distances over the same type of the road at the same speed [9]. In reality over-the-road transport vibration environments are constantly changing due to factors such as the road surface and vehicle speed.
These factors will vary the intensity of the vibrations observed by the vehicle. Because these factors are changing, the statistical characteristics are also changing, meaning the vibration is no longer stationary, but can be described as non-stationary [10]. Real Vibrations located in Victoria, Australia has put forward the hypothesis that non-stationary (and non-Gaussian) random vibrations can be decomposed into independent random Gaussian elements with varying amplitudes, standard deviations, and durations [9].

Over-the-road vibration data was recorded from a steel spring truck traveling from Anderson, $\mathrm{SC}$ to Dallas, TX. The vibration data was analyzed based on the above mentioned analysis techniques and simulations were performed. Previous research has evaluated simulated products and theory, but has not provided experimental testing with packaged products. Where this research is unique is that it evaluates the simulation techniques using actual packaged products, and by aid of damage assessment tools, determines how well the techniques correlated with actual field study results.

\subsection{MATERIALS AND METHODS}

A Shock and Vibration Environment Recorder (SAVER ${ }^{\mathrm{TM}}$ ) (Lansmont Corp., Monterey, CA) model 9X30 was used in this study. These data recorders use an internal triaxial accelerometer to measure vibration levels. The data recorder was rigidly mounted inside at the rear of the trailer. Figure 1 (page 4) illustrates the location of the data recorder.

The data recorder was rigidly attached to a fully loaded 53' trailer. The trailer used had a steel spring suspension, and when fully loaded had an overall payload weight of $16,800 \mathrm{lbs}$. The trailer was fully loaded throughout the entire trip recorded. The data recorder was turned on prior to the 


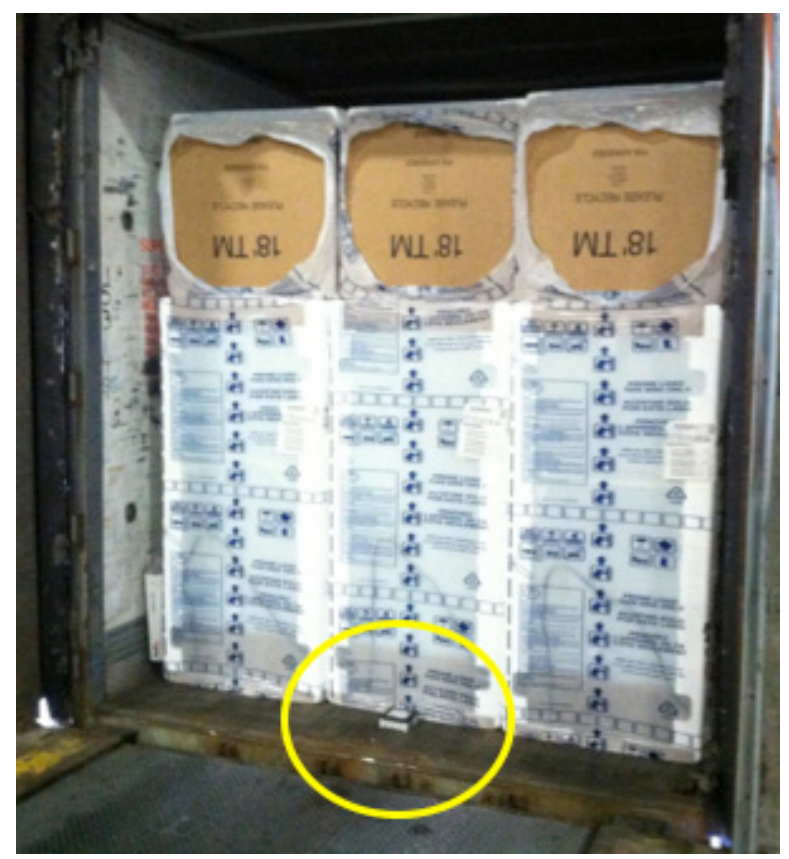

Figure 1. SAVER ${ }^{\mathrm{TM}} 9 X 30$ location inside rear of trailer

trailer leaving the warehouse in Anderson, SC and was turned off when it arrived at the distribution center near Dallas, TX.

The data recorder was programmed to record and analyze vibration using both signal and timer triggered data collecting methods. Signal triggered data refers to the data recorded during an event in which the intensity exceeds a preset threshold. Timer trigger data refers to the data recorder "waking up" at a preset frequency and recording for a preset duration.

Although both data collecting methods were employed for this study, only the signal triggered data was used for analysis during this study. This is due to the very low trigger threshold employed. The low trigger threshold allowed for the data recorder to record the vehicle motion throughout the entire trip. Traditional data recording methods can be skewed due to the level of the trigger threshold. Traditional methods (trigger threshold of $0.5 \mathrm{G}$ ) result in a higher overall Grms level, but for this study, having a low trigger threshold enabled all vibration events of importance to be recorded using only the signal triggered data. By setting a low trigger threshold, the overall Grms level is representative of the entire trip while the vehicle was in motion. The following were the recording parameters used in the collection of the signal triggered data:

Signal Triggered Data

- Event Trigger Threshold: $0.10 \mathrm{G}$

- Sample Rate: 1000 Samples/Sec

- Record Time: $2.048 \mathrm{sec}$.

- Signal Pre-Trigger: $20 \%$

\subsection{Packaged Products and Damage Assessment}

The test units used for this study were a topmount refrigerator, electric hedge trimmer, and gas powered pressure washer. Prior to testing, the units were visually inspected for damage. Any damage was noted and the units were repackaged for testing. The units were positioned on a $60 \times 60$ in. Lansmont Corporation servo-hydraulic vibration table as they would be for actual transit.

Two refrigerators were positioned upright on the vibration table, and one refrigerator was placed in the prone position on top. For each vibration test, the prone and one upright unit were used as test units and the second upright unit as a dummy unit. New test units were used for each vibration test method evaluated.

In order to evaluate the refrigerators at the conclusion of each vibration test simulation, a damage assessment protocol was used. The part used for 
Table 1. Damage assessment protocol for refrigerators

\begin{tabular}{|c|c|c|}
\hline Assessment Grade & Brief Description & Amount of Abrasion \\
\hline 1 & No visible damage to unit & None \\
\hline 2 & Minor paint abrasion & $\leq 0.5 \mathrm{in}$. \\
\hline 3 & Major paint abrasion and denting & $\geq 1.0 \mathrm{in}$. \\
\hline 4 & Structural damage to unit & N/A \\
\hline
\end{tabular}

Table 2. Damage assessment protocol for hedge trimmer

\begin{tabular}{|c|c|c|}
\hline Assessment Grade & Brief Description & Amount of Abrasion \\
\hline 1 & No visible damage to unit or package & None \\
\hline 2 & Minor abrasion to unit and container & $\leq 0.5 \mathrm{in}$. \\
\hline 3 & Major abrasion to unit and container & $\geq 1.0 \mathrm{in}$ \\
\hline 4 & Structural damage to unit & N/A \\
\hline
\end{tabular}

Table 3. Damage assessment protocol for pressure washer

\begin{tabular}{|c|c|c|}
\hline Assessment Grade & Brief Description & Amount of Abrasion \\
\hline 1 & No visible damage to unit & None \\
\hline 2 & Minor paint abrasion & $\leq 0.5 \mathrm{in}$. \\
\hline 3 & Major paint abrasion and denting & $\geq 1.0 \mathrm{in}$. \\
\hline 4 & Structural damage to unit & N/A \\
\hline
\end{tabular}

this evaluation was the internal ladder in which the cantilever shelves were placed. The damage assessment focused on the finish to the painted ladders and cantilever shelves. Table 1 displays the damage assessment used during the evaluation. Assessment grades being rated as 1 or 2 closely correlated with actual field study results of the refrigerators. Assessment grades of 3 or 4 do not correlate with actual field study results.

The hedge trimmer was packaged inside a single-wall corrugated container. A single container 
was placed on the vibration table in proper shipping orientation for the vibration test. In order to evaluate the hedge trimmers, a damage assessment protocol was used. For this evaluation, both the package and the product were evaluated. Table 2 displays the damage assessment used during the evaluation. Assessment grades being rated as 1 or 2 closely correlated with actual field study results of the hedge trimmers. Assessment grades of 3 or 4 do not correlate with actual field study results.

The pressure washer was packaged inside a double-wall corrugated container. A single container was placed on the vibration table in proper shipping orientation for the vibration test. In order to evaluate the pressure washer, a damage assessment protocol was used. For this evaluation, both the package and the product were evaluated. Table 3 displays the damage assessment used during the evaluation. Assessment grades being rated as 1 or 2 closely correlated with actual field study results of the pressure washer. Assessment grades of 3 or 4 do not correlate with actual field study results.

\subsection{RESULTS AND DISCUSSION}

SaverXware programming software was used for initial analysis of the data recorded. For all analysis methods, only the vertical axis data was evaluated as it resulted in the greatest overall vibration intensity of all axes recorded, and was the only one that could be simulated. The range for the signal trigger events captured during the shipment was from 0.01 RMS G to 1.729 RMS G. The duration of recording was 8 hours and 9 minutes.

The following sections will discuss and review how the data was analyzed for each of the vibration methods used for this study.

\subsection{Time Compression (Accelerated) Vibration}

SaverXware programming software was used to develop the average PSD profile. The PSD profile generated had an average overall Grms level of 0.195 . To perform the time compressed vibration test, the average overall Grms and the duration of the shipment were used in the calculation. Also needed was the test time (in hours), which was 3 hours. The test time of 3 hours is a result of not exceeding the 5:1 ratio. The below calculation shows how the overall Grms for the time compressed vibration test was determined:

$$
0.44 \mathrm{Grms}=0.195 \mathrm{Grms} \sqrt{\frac{15 H r s}{3 H r s}}
$$

The overall Grms for the time compressed vibration test was calculated to be $0.44 \mathrm{Grms}$. The breakpoints for this PSD profile were loaded into the vibration controller and used to drive the vibration table for 3 hours.

At the completion of the vibration simulation, the test units were visually inspected for damage. During the inspection it was noted there was significant abrasion and scuffing to the painted surfaces of the packaged products. The results from this showed the time compressed vibration test did not correlate with the results of the field shipments. As a result of the inspection, the products evaluated during this simulation were graded as a 3 .

\subsection{Constructed Probability Analysis}

SaverXware programming software was used to build the report used for the test protocol. Table 4 shows the results of the constructed probability analysis reported by the SaverXware software. Also, as part of the summary analysis, separate 
Table 4. Constructed probability analysis report

\begin{tabular}{|l|l|l|l|l|}
\hline Probability Spectra & Overall Grms & Event Count & Test Duration (H:M) & Stroke (in.) \\
\hline $\mathbf{5 0 \%}$ (Average) & 0.195 & 7062 & $04: 01$ & 1.42 \\
\hline $\mathbf{8 0 \%}$ at or Below & 0.210 & 2515 & $01: 26$ & 1.45 \\
\hline $\mathbf{9 0 \%}$ at or Below & 0.301 & 4425 & $02: 31$ & 2.56 \\
\hline $\mathbf{9 5 \%}$ at or Below & 0.397 & 253 & $00: 09$ & 3.67 \\
\hline $\mathbf{9 9 \%}$ at or Below & 0.654 & 59 & $00: 02$ & 3.89 \\
\hline
\end{tabular}

Table 5. Constructed probability analysis reduced test setup and results

\begin{tabular}{|l|l|l|l|l|}
\hline Probability Spectra & Test Duration (H:M) & Refrigerator & Hedge Trimmer & Pressure Washer \\
\hline $\mathbf{5 0 \%} \rightarrow \mathbf{9 9} \%$ & $08: 09$ & Correlated & Correlated & Correlated \\
\hline $\mathbf{8 0} \% \rightarrow \mathbf{9 9 \%}$ & $04: 08$ & Correlated & Correlated & Correlated \\
\hline $\mathbf{9 0 \%} \rightarrow \mathbf{9 9 \%}$ & $02: 42$ & Correlated & Correlated & Correlated \\
\hline $\mathbf{9 5 \%} \rightarrow \mathbf{9 9 \%}$ & $00: 11$ & No Correlation & No Correlation & No Correlation \\
\hline $\mathbf{9 9} \%$ & $00: 02$ & No Correlation & No Correlation & No Correlation \\
\hline
\end{tabular}

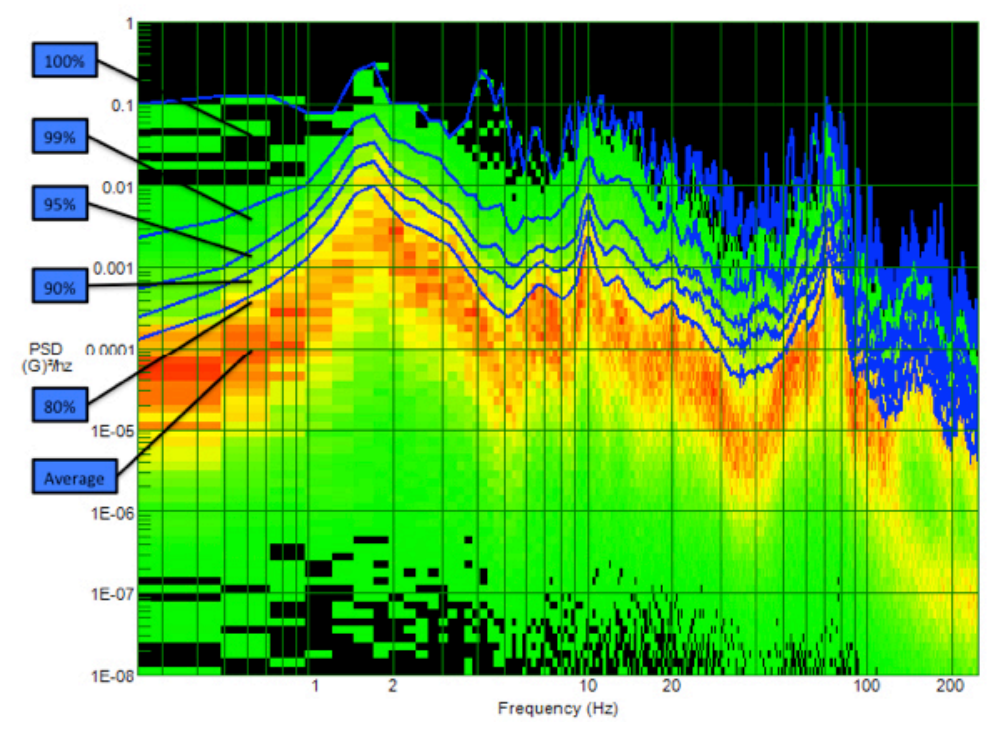

Figure 2. PSD profiles generated from constructed probability analysis

PSD profiles were generated for each of the percentile ranges. Figure 2 illustrates the separate PSD profiles graphically overlaid on one graph.

The vibration controller was loaded with the various PSD profiles which were to be run from the $50 \%$ to the $99 \%$ in sequential order. In order to reduce the test time of the vibration simulations, one or more PSD profiles were eliminated from the test queue. For example, the $50 \%$ to the $99 \%$ uses all of the PSD's and the corresponding durations, whereas for the $80 \%$ to the $99 \%$ test simula- 
tion, the $50 \%$ (average) was not used as part of the simulation. This allows for the total test time to be reduced by using the data collected from the study, and potentially eliminating lower intensity vibration and only focusing on the vibration intensities of concern. Table 5 displays how the vibration simulations were conducted and provides whether or not the simulation correlated with the field data.

The results from the Constructed Probability Analysis show it is possible to reduce the test time of the vibration simulation, while still correlating with the results from the field data. The 50\% to the $99 \%, 80 \%$ to the $99 \%$, and the $90 \%$ to the $99 \%$ all correlated well with the results from the field study. For this study it showed it was possible to replicate the 15 hour shipment by testing for 2 hours and 42 minutes using the $90 \%$ to the $99 \%$ spectrums and durations.

The $95 \%$ to the $99 \%$ and just the $99 \%$ test simulations did not correlate with the field samples. No visible damage was noted to the units as a result of the simulation resulting in no comparisons to be made to the field data. This was largely believed to be as a result of the durations of these simulations.

\subsection{Synthesis of Non-stationary and Non- Gaussian}

In order to analyze the vibration data by this method, a Statistical Vibration Synthesizer (SVS) was obtained from Real Vibrations. The individual vibration events recorded were loaded into the SVS and the software generated the RMS distribution. For traditional vibration testing, the PSD profiles are Gaussian and operate at the specified Grms level for $100 \%$ of the time. The major difference with this method in comparison to traditional PSD profiles is the SVS generates the RMS distribution that is to be followed. This is an oversimplification, but the SVS analyzes the vibration events and groups the events by RMS levels into bins. Each bin is also given a dose or percentage at which it will operate over the length of the test. These bins are then collectively used to operate the vibration table at $100 \%$. Therefore the vibration test is no longer operating at a single Grms level as with traditional PSD testing, but operating at various RMS levels (Figure 3).

After the data was analyzed by the SVS, the resulting PSD profile and PDF were used to drive
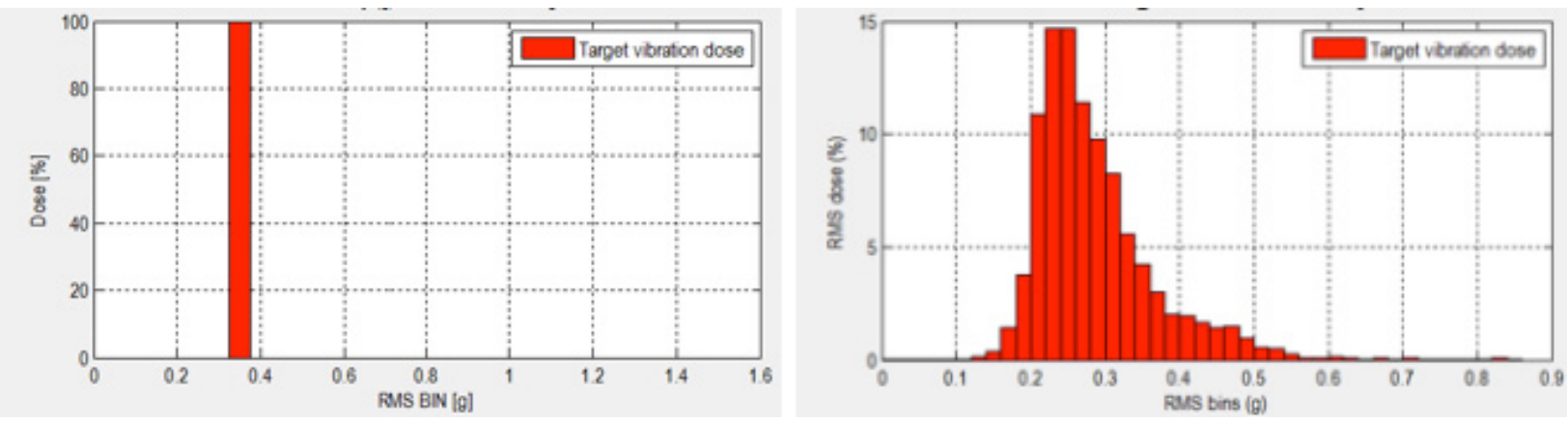

Figure 3. Comparison of vibration dose: Traditional Method (left) with SVS Method (right) 
Table 6. Non-Stationary and Non-Gaussian test setup and results

\begin{tabular}{|l|l|l|l|l|}
\hline Overall Grms & Test Duration (H:M) & Refrigerator & Hedge Trimmer & Pressure Washer \\
\hline $\mathbf{8 0 . 1 9 5}$ & $08: 09$ & Correlated & Correlated & Correlated \\
\hline $\mathbf{> 0 . 2 1 0}$ & $04: 08$ & Correlated & Correlated & Correlated \\
\hline $\mathbf{> 0 . 3 0 1}$ & $02: 42$ & Correlated & Correlated & Correlated \\
\hline $\mathbf{> 0 . 3 9 7}$ & $00: 11$ & No Correlation & No Correlation & No Correlation \\
\hline $\mathbf{8} \mathbf{0 . 6 5 4}$ & $00: 02$ & No Correlation & No Correlation & No Correlation \\
\hline
\end{tabular}

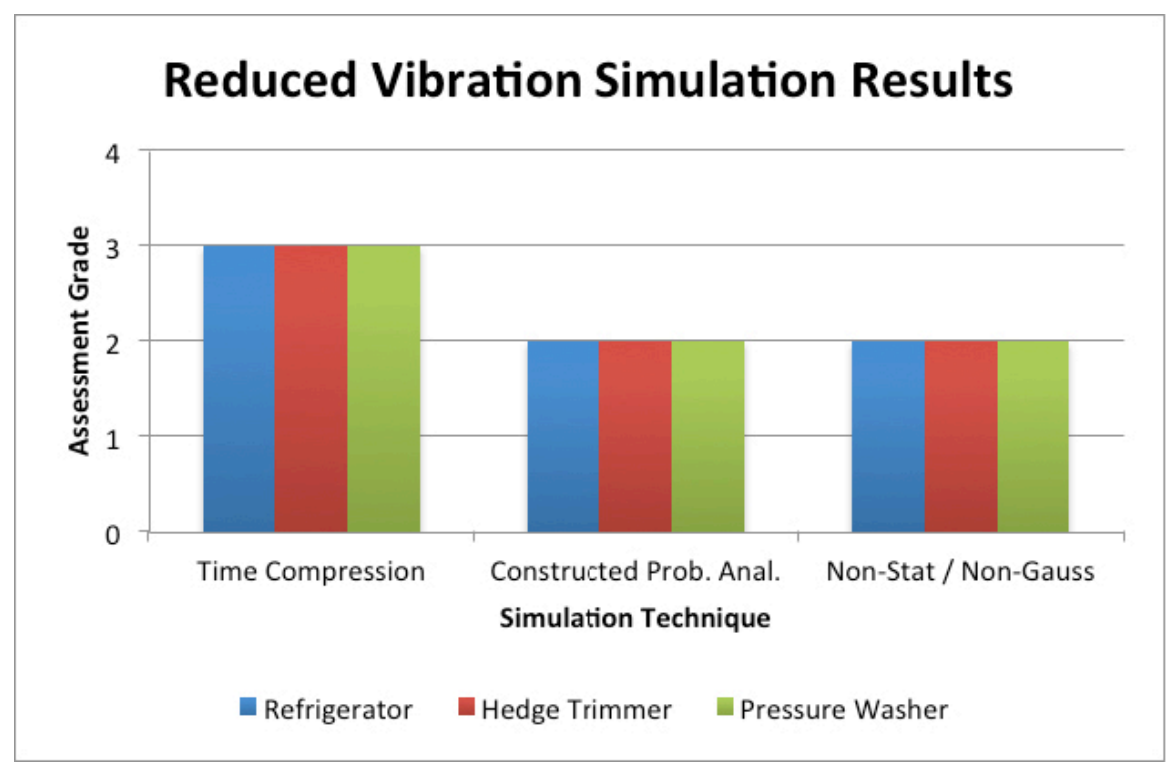

Figure 4. Summary of test results for reduced vibration simulation techniques

the vibration table. Adjusting which RMS bins are used by the SVS to generate the simulation can reduce the test time. This is accomplished through de-selecting the RMS bins used to for the simulation. For example, de-selecting all RMS bins below 0.21 , allows only the data greater than this to be used in the simulation. Table 6 displays the Grms levels and durations used for these simulations as well as the test results.

Similarly to the Constructive Probability Analysis, the results from the Non-stationary and NonGaussian method showed it was possible to reduce the vibration test time while still replicating the field data.

Figure 4 provides a graphical summary of the assessment grades given for each of the vibration simulation techniques evaluated. The Time Compression (Accelerated) vibration simulation resulted in all packaged products receiving an assessment grade of 3 meaning the results did not correlate with the field samples evaluated. Both the Constructed Probability Analysis and the NonStationary and Non-Gaussian vibration simulations were able to show correlation with field 
samples while reducing the vibration test time. The samples for both the Constructed Probability Analysis and the Non-Stationary and Non-Gaussian were graded as a 2 .

\subsection{Implementation Challenge}

Recording all "in-motion" vehicle vibration presents challenges of very large data files and capturing the total duration of the selected trip. Based on the data recorder and method employed for this study, the duration of trip that could be recorded was 8 hours and 9 minutes. The duration of the actual trip for this study was 15 hours. By reducing the sample rate and sample size, "in-motion" vehicle vibration measurements could be increased to record longer duration trips. Also, based on the 'Max Overwrite' setting it could be assumed all vibration events of importance were captured as the trigger threshold was set at $0.1 \mathrm{G}$ meaning for this study the other 7 hours not captured in the analysis were at or below $0.1 \mathrm{G}$.

\subsection{CONCLUSIONS}

Examined were three vibration techniques for reducing the vibration simulation time. Results indicate both the Constructed Probability Analysis and the Non-Stationary and Non-Gaussian methods can in fact reduce the test time while still correlating with actual field shipments of the packaged products. The Time Compression method for reducing the test duration increased the amount of damage and did not reproduce results of actual field shipments of these packaged products. Realized were challenges encountered when implementing the different methods, including a change in data acquisition methodology which resulted in limited record times based on available data recorder settings.

\section{ACKNOWLEDGEMENTS}

Portions of this article were previously published and presented at the ISTA International Transport Packaging Forum ${ }^{\mathrm{TM}}$ (April 2014).

\section{REFERENCES}

[1] McKinley, A.H. (2004) Transport Packaging. $2^{\text {nd }} E d$. Institute of Packaging Professionals.

[2] Griffiths, K., Shires, D., White, W., Keogh, P. S. and Hicks, B. J. (2013), Correlation Study Using Scuffing Damage to Investigate Improved Simulation Techniques for Packaging Vibration Testing. Packag. Technol. Sci., 26: 373-383. doi: 10.1002/ pts. 1987 Link

[3] Kipp, W. (2001) "Accelerated Random Vibration with Time-History Shock" IoPP 2001 Annual Membership Meeting, San Jose, CA., 2001 Link

[4] Bernad, C., Laspalas, A., González, D., Núñez, J. L. and Buil, F. (2011), Transport vibration laboratory simulation: On the necessity of multiaxis testing. Packag. Technol. Sci., 24: 1-14. doi: 10.1002/pts.913 Link

[5] Dunno, K. and Batt, G. (2013) "Experimental Comparison of Vibration Simulation Techniques" ISTA Transpack Forum, March 2013.

[6] ASTM International (2009) ASTM D4169 Standard Practice for Performance Testing of Shipping Containers and Systems: ASTM: West Conshohocken, PA, 2009. Link

[7] ISTA Resource Book (2013) ISTA: East Lansing, MI, 2013.

[8] Root, D. (2010) "Constructed Probability, Applying Analysis Results in the Lab in Pursuit of Improved Damage Correlation" ISTA International Transport Packaging Forum, Orlando, FL, 2010. 